\title{
An analysis of consultations with the crowd doctors at Glasgow Celtic football club, season 1999-2000
}

M Crawford, J Donnelly, J Gordon, R MacCallum, I MacDonald, M McNeill, N Mulhearn, S Tilston, G West

Hairmyres Hospital, East Kilbride, Scotland, UK M Crawford

The Royal Infirmary, Lauriston Place, Edinburgh EH3 9YO, Scotland, UK

J Donnelly

Accident and

Emergency

Department, The

Royal Alexandra

Hospital, Paisley

PA2 9PN, Scotland, UK

J Gordon

Accident and

Emergency

Department, Victoria

Infirmary, Glasgow

G42 9TY, Scotland, UK

$\mathrm{R}$ MacCallum

162 Nithsdale Road, Glasgow G41 5RU, Scotland, UK

I MacDonald

Glasgow Royal

Infirmary, Glasgow

G4 0ST, Scotland, UK

$\mathrm{M} \mathrm{McNeill}$

General Medicine, Falkirk and District Royal Infirmary, Majors Loan, Falkirk FK1 5QE, Scotland, UK

N Mulhearn

Department of Dermatology,

Monklands Hospital,

Monkscourt Avenue,

Airdrie ML6 0JS,

Scotland, UK

$S$ Tilston

Woodside Health Centre, Barr St, Glasgow G20 4RL, UK

G West

Correspondence to: Dr MacDonald, 5 Merrylee Road, Glasgow G43 2SH, Scotland, UK

ian_macdonald31@hotmail.com

Accepted 26 April 2001

\begin{abstract}
Objective-To analyse all clinical presentations to the crowd doctors at Scotland's largest football stadium over the course of one complete season.

Methods-A standard clinical record form was used to document all consultations with the crowd doctors including treatment and subsequent referrals. The relevance of alcohol consumption was assessed.

Results-A total of 127 casualties were seen at 26 matches, a mean of 4.88 per match. Twenty casualties were transferred to hospital, including one successfully defibrillated after a cardiac arrest. Alcohol excess was a major contributing factor in 26 cases.

Conclusions-The workload of the crowd doctors was very variable and diverse. The social problem of excessive alcohol consumption contributed considerably to the workload. The provision of medical facilities at football grounds means that attendance there is now one of the least adverse circumstances in which to have a cardiac arrest. The study confirmed previous impressions that more casualties are seen at high profile matches.

(Br F Sports Med 2001;35:245-250)
\end{abstract}

Keywords: crowd doctor; major sporting event; football grounds; Gibson report

There have been three major disasters in one generation at British football grounds: at Ibrox Park in 1971 causing 66 deaths, at Bradford in 1985 causing 40 deaths, and most recently at the Hillsborough Stadium in Sheffield in 1989 causing 95 deaths. In the aftermath of these tragedies, there have been substantial changes in the requirements for the provision of safety at football grounds. These followed recommendations in the Wheatley report ${ }^{1}$ published after the Ibrox disaster, and then more comprehensively in the Taylor report ${ }^{2}$ into the Hillsborough Stadium disaster. In conjunction with the Taylor report, the Gibson report ${ }^{3}$ made specific recommendations for the provision of medical care at football grounds. These recommendations have been described in previous articles on this subject by other authors. ${ }^{45}$

A central recommendation of the Gibson report was the responsibility for clubs to provide suitably trained and equipped crowd doctors. Attention has recently been drawn to the incomplete implementation of this recommendation in Scotland. ${ }^{4}$ The authors are the crowd doctors for Glasgow Celtic, one of Scotland's leading football clubs. Since the development of its new enlarged stadium, Celtic now require three crowd doctors for each game. These doctors are drawn from the disciplines of accident and emergency medicine, anaesthetics, and general practice, ensuring the provision of a good mixture of skills. There is a consultant anaesthetist at every game, ensuring that the highest level of resuscitation skills is always available. The general practitioners involved have obtained Advanced Life Support Provider certification from the UK Resuscitation Council. The accident and emergency consultant and one general practitioner have the Major Incident Medical Management and Support (MIMMS) certificate. At virtually every game there is a MIMMS trained doctor on duty.

The crowd doctors are in radio contact with the Scottish Ambulance Service control point and work from the trackside or upper tier of the stadium alongside the other medical care providers. There are four ambulance crews (one paramedic and one technician) and an ambulance incident officer at every game, with the Emergency Support Unit vehicle also in attendance. There are in addition another two ambulance paramedics and two technicians whose responsibility is the safe removal of injured players from the field of play. Finally there are 50-60 voluntary first aid workers from the St Andrew's Ambulance Association, whose skills and commitment are indispensable.

An essential responsibility of the crowd doctors is to manage the initial medical response to a major incident. Celtic has a major incident plan, which is regularly updated, most recently after a large tabletop exercise at the stadium last year. This was attended by the crowd doctors along with representatives of the club, the emergency services, and local authority services. It is hoped, however, that the likelihood of such an incident has been reduced substantially by measures such as all seated stadia, all ticket games, and improved stewarding.

The routine work of the crowd doctors is to treat the casualties referred to them by first aid or ambulance service workers. This is a study of all such consultations during the season 1999-2000.

\section{Methods}

An audit form (fig 1) was used to document every consultation with the crowd doctors during the season 1999-2000. In addition to the clinical notes, information was obtained about 


$$
\text { GAME: CELTIC V }
$$

DATE:

NAME:

AGE:

RESIDENCE (e.g. Glasgow, Aberdeen)

CLINICAL NOTES:-
DOCTOR: 
Table 2 List of casualties seen at Glasgow Celtic Football Club in the 1999-2000 season

\begin{tabular}{|c|c|c|c|c|c|c|c|}
\hline Date & Opposition & Age & Sex & Clinical information & $\begin{array}{l}\text { Relevance } \\
\text { of alcohol }\end{array}$ & $\begin{array}{l}\text { Timing in } \\
\text { relation to game }\end{array}$ & Treatment/outcome \\
\hline 24/7/99 & Leeds Utd & $\begin{array}{l}29 \\
10 \\
19 \\
30\end{array}$ & $\begin{array}{l}\text { Female } \\
\text { Male } \\
\text { Female } \\
\text { Male }\end{array}$ & $\begin{array}{l}\text { Collapse, inebriated } \\
\text { Viral illness } \\
\text { Collapse } \\
\text { Foreign body }(\mathrm{FB}) \text { in ear }\end{array}$ & $\begin{array}{l}\text { Major } \\
\text { None } \\
\text { None } \\
\text { None }\end{array}$ & $\begin{array}{l}\text { Related } \\
\text { Unrelated } \\
\text { Related } \\
\text { Related }\end{array}$ & $\begin{array}{l}\text { Discharged } \\
\text { Paracetamol } \\
\text { Reassured } \\
\text { FB removed }\end{array}$ \\
\hline $27 / 7 / 99$ & Newcastle Utd & $\begin{array}{l}34 \\
28 \\
41 \\
17\end{array}$ & $\begin{array}{l}\text { Male } \\
\text { Male } \\
\text { Female } \\
\text { Female }\end{array}$ & $\begin{array}{l}\text { Collapse, sweaty; previous MI } \\
\text { Epileptic ; ?aura } \\
\text { Finger injury } \\
\text { Wrist sprain }\end{array}$ & $\begin{array}{l}\text { Minor } \\
\text { Major } \\
\text { None } \\
\text { None }\end{array}$ & $\begin{array}{l}\text { Related } \\
\text { Related } \\
\text { Related } \\
\text { Related }\end{array}$ & $\begin{array}{l}\text { Aspirin, hospital } \\
\text { Observation, reassured } \\
\text { Reassured } \\
\text { Reassured }\end{array}$ \\
\hline 7/8/99 & St Johnstone & 22 & Male & Toothache & None & Unrelated & Paracetamol \\
\hline 26/8/99 & Cwmbran Town & $\begin{array}{l}18 \\
63 \\
19\end{array}$ & $\begin{array}{l}\text { Male } \\
\text { Male } \\
\text { Male }\end{array}$ & $\begin{array}{l}\text { Nausea, dizziness } \\
\text { Left knee gave way } \\
\text { Dog bite on thigh }\end{array}$ & $\begin{array}{l}\text { None } \\
\text { None } \\
\text { None }\end{array}$ & $\begin{array}{l}\text { Related } \\
\text { Related } \\
\text { Related }\end{array}$ & $\begin{array}{l}\text { Reassurance } \\
\text { Left knee locked, hospital } \\
\text { Abrasions cleaned }\end{array}$ \\
\hline 29/8/99 & Hearts & $\begin{array}{l}34 \\
25 \\
38\end{array}$ & $\begin{array}{l}\text { Male } \\
\text { Male } \\
\text { Male }\end{array}$ & $\begin{array}{l}\text { Migraine } \\
\text { Swallowed wasp and vomited } \\
\text { Anxiety, intoxicated }\end{array}$ & $\begin{array}{l}\text { None } \\
\text { Minor } \\
\text { Major }\end{array}$ & $\begin{array}{l}\text { Related } \\
\text { Related } \\
\text { Related }\end{array}$ & $\begin{array}{l}\text { Paracetamol } \\
\text { Observation, discharged } \\
\text { Observation, reassurance }\end{array}$ \\
\hline $16 / 9 / 99$ & Tel Aviv & $\begin{array}{l}41 \\
22\end{array}$ & $\begin{array}{l}\text { Female } \\
\text { Female }\end{array}$ & $\begin{array}{l}\text { Hot splash to left eye } \\
\text { Pregnant. Brief abdominal pain }\end{array}$ & $\begin{array}{l}\text { None } \\
\text { None }\end{array}$ & $\begin{array}{l}\text { Related } \\
\text { Related }\end{array}$ & $\begin{array}{l}\text { Eye bath } \\
\text { Examined and reassured }\end{array}$ \\
\hline $16 / 10 / 99$ & Aberdeen & $\begin{array}{l}50 \\
30 \\
31 \\
51 \\
15\end{array}$ & $\begin{array}{l}\text { Male } \\
\text { Male } \\
\text { Male } \\
\text { Male } \\
\text { Male }\end{array}$ & $\begin{array}{l}\text { Angina } \\
\text { Headache } \\
\text { Bereavement } \\
\text { Injury to knee } \\
\text { Alcohol and ecstasy ingestion }\end{array}$ & $\begin{array}{l}\text { Minor } \\
\text { None } \\
\text { None } \\
\text { None } \\
\text { Major }\end{array}$ & $\begin{array}{l}\text { Related } \\
\text { Related } \\
\text { Unrelated } \\
\text { Related } \\
\text { Related }\end{array}$ & $\begin{array}{l}\text { Nitrolingual spray } \\
\text { Paracetamol } \\
\text { Advice } \\
\text { Reassured } \\
\text { Hospital }\end{array}$ \\
\hline $27 / 10 / 99$ & Motherwell & $\begin{array}{l}13 \\
73 \\
23 \\
42 \\
19 \\
16 \\
9\end{array}$ & $\begin{array}{l}\text { Male } \\
\text { Male } \\
\text { Male } \\
\text { Male } \\
\text { Male } \\
\text { Female } \\
\text { Male }\end{array}$ & $\begin{array}{l}\text { ?Viral illness } \\
\text { Brief chest pain. Previous MIs } \\
\text { Assaulted outside ground. Scalp laceration } \\
\text { Collapse. Chest pain } \\
\text { Asthma } \\
\text { Minor burns to hand } \\
\text { Minor head injury }\end{array}$ & $\begin{array}{l}\text { None } \\
\text { Major } \\
\text { Minor } \\
\text { Major } \\
\text { Minor } \\
\text { None } \\
\text { None }\end{array}$ & $\begin{array}{l}\text { Related } \\
\text { Related } \\
\text { Related } \\
\text { Related } \\
\text { Related } \\
\text { Related } \\
\text { Related }\end{array}$ & $\begin{array}{l}\text { Reassurance } \\
\text { Examined and reassured } \\
\text { Hospital for suturing } \\
\text { Hospital } \\
\text { Salbutamol inhaler } \\
\text { Reassured } \\
\text { Reassured }\end{array}$ \\
\hline $30 / 10 / 99$ & Kilmarnock & $\begin{array}{l}30 \\
71 \\
71 \\
2 \\
52 \\
56\end{array}$ & $\begin{array}{l}\text { Male } \\
\text { Male } \\
\text { Male } \\
\text { Male } \\
\text { Male } \\
\text { Male }\end{array}$ & $\begin{array}{l}\text { Injury to right knee-swollen and locked } \\
\text { Head injury } \\
\text { Asthma } \\
\text { Injury to left forearm ?greenstick fracture } \\
\text { Hypertension, confusion. TIA } \\
\text { Angina }\end{array}$ & $\begin{array}{l}\text { None } \\
\text { None } \\
\text { None } \\
\text { None } \\
\text { None } \\
\text { None }\end{array}$ & $\begin{array}{l}\text { Related } \\
\text { Related } \\
\text { Related } \\
\text { Related } \\
\text { Related } \\
\text { Related }\end{array}$ & $\begin{array}{l}\text { Hospital } \\
\text { Observation, then discharged } \\
\text { Salbutamol inhaler } \\
\text { Father to take to A\&E } \\
\text { Hospital } \\
\text { Nitrolingual spray }\end{array}$ \\
\hline $4 / 11 / 99$ & Lyon & $\begin{array}{l}26 \\
16 \\
19 \\
14 \\
46 \\
22 \\
18\end{array}$ & $\begin{array}{l}\text { Male } \\
\text { Female } \\
\text { Male } \\
\text { Male } \\
\text { Female } \\
\text { Female } \\
\text { Male }\end{array}$ & $\begin{array}{l}\text { Fall: head, face and shoulder injury } \\
\text { Burn to right forearm } \\
\text { Severe facial injury } \\
\text { Inebriated } \\
\text { Migraine } \\
\text { Fall: injury to coccyx } \\
\text { Grand mal, post ictal }\end{array}$ & $\begin{array}{l}\text { Major } \\
\text { None } \\
\text { Major } \\
\text { Major } \\
\text { None } \\
\text { None } \\
\text { None }\end{array}$ & $\begin{array}{l}\text { Related } \\
\text { Related } \\
\text { Related } \\
\text { Related } \\
\text { Related } \\
\text { Related } \\
\text { Related }\end{array}$ & $\begin{array}{l}\text { Hospital } \\
\text { Cold water } \\
\text { Hospital } \\
\text { Hospital } \\
\text { Paracetamol } \\
\text { Paracetamol } \\
\text { Hospital }\end{array}$ \\
\hline $1 / 12 / 99$ & Dundee & $\begin{array}{l}12 \\
38\end{array}$ & $\begin{array}{l}\text { Female } \\
\text { Female }\end{array}$ & $\begin{array}{l}\text { Abdominal pain } \\
\text { Asthma }\end{array}$ & $\begin{array}{l}\text { None } \\
\text { None }\end{array}$ & $\begin{array}{l}\text { Unrelated } \\
\text { Related }\end{array}$ & $\begin{array}{l}\text { Reassured } \\
\text { Salbutamol inhaler }\end{array}$ \\
\hline $4 / 12 / 99$ & Hibernian & $\begin{array}{l}29 \\
38 \\
16\end{array}$ & $\begin{array}{l}\text { Male } \\
\text { Male } \\
\text { Male }\end{array}$ & $\begin{array}{l}\text { ?Scaphoid fracture } \\
\text { Vasovagal episode } \\
\text { Torticollis }\end{array}$ & $\begin{array}{l}\text { None } \\
\text { None } \\
\text { None }\end{array}$ & $\begin{array}{l}\text { Related } \\
\text { Related } \\
\text { Unrelated }\end{array}$ & $\begin{array}{l}\text { Advised to attend } \mathrm{A} \& \mathrm{E} \\
\text { Reassured } \\
\text { Cocodamol }\end{array}$ \\
\hline $18 / 12 / 99$ & Dundee Utd & $\begin{array}{l}24 \\
34 \\
16 \\
40 \\
38 \\
16 \\
20 \\
41\end{array}$ & $\begin{array}{l}\text { Male } \\
\text { Male } \\
\text { Male } \\
\text { Male } \\
\text { Male } \\
\text { Male } \\
\text { Male } \\
\text { Female }\end{array}$ & $\begin{array}{l}\text { Shoulder injury } \\
\text { Collapse, intoxicated } \\
\text { Viral illness } \\
\text { Minor scalp laceration, struck by coin } \\
\text { Intoxicated; refused entry to ground } \\
\text { Viral illness } \\
\text { Collapse, faked seizure } \\
\text { Superficial scalp scald }\end{array}$ & $\begin{array}{l}\text { None } \\
\text { Major } \\
\text { None } \\
\text { None } \\
\text { Major } \\
\text { None } \\
\text { Major } \\
\text { None }\end{array}$ & $\begin{array}{l}\text { Unrelated } \\
\text { Related } \\
\text { Unrelated } \\
\text { Related } \\
\text { Related } \\
\text { Unrelated } \\
\text { Related } \\
\text { Related }\end{array}$ & $\begin{array}{l}\text { Reassured } \\
\text { Left in police custody } \\
\text { Reassured } \\
\text { Wound cleaned } \\
\text { Examined } \\
\text { Paracetamol } \\
\text { Left in police custody } \\
\text { No treatment }\end{array}$ \\
\hline $27 / 12 / 99$ & Rangers & $\begin{array}{l}24 \\
12 \\
29 \\
15 \\
36 \\
55 \\
18 \\
38 \\
31 \\
21\end{array}$ & $\begin{array}{l}\text { Female } \\
\text { Male } \\
\text { Male } \\
\text { Male } \\
\text { Male } \\
\text { Female } \\
\text { Male } \\
\text { Male } \\
\text { Male } \\
\text { Male }\end{array}$ & $\begin{array}{l}\text { Migraine } \\
\text { Bruise to forehead - struck by coin } \\
\text { Injury to left knee } \\
\text { Headache } \\
\text { Dizziness } \\
\text { Asthma } \\
\text { Vomiting } \\
\text { ?Corneal abrasion } \\
\text { Facial wound } \\
\text { URTI }\end{array}$ & $\begin{array}{l}\text { None } \\
\text { None } \\
\text { None } \\
\text { None } \\
\text { Major } \\
\text { None } \\
\text { Major } \\
\text { None } \\
\text { None } \\
\text { None }\end{array}$ & $\begin{array}{l}\text { Related } \\
\text { Related } \\
\text { Related } \\
\text { Related } \\
\text { Related } \\
\text { Related } \\
\text { Related } \\
\text { Related } \\
\text { Related } \\
\text { Unrelated }\end{array}$ & $\begin{array}{l}\text { Paracetamol } \\
\text { Ice } \\
\text { Ice, tubigrip, paracetamol } \\
\text { Paracetamol } \\
\text { Reassurance } \\
\text { Salbutamol inhaler } \\
\text { Examined and reassured } \\
\text { Advised to attend A\&E after match } \\
\text { Advised to attend A\&E after match } \\
\text { Reassured }\end{array}$ \\
\hline
\end{tabular}

Table 2 continued on next page. 
Table 2 continued

\begin{tabular}{|c|c|c|c|c|c|c|c|}
\hline Date & Opposition & Age & Sex & Clinical information & $\begin{array}{l}\text { Relevance } \\
\text { of alcohol }\end{array}$ & $\begin{array}{l}\text { Timing in } \\
\text { relation to game }\end{array}$ & Treatment/outcome \\
\hline $1 / 2 / / 00$ & Bayern Munich & $\begin{array}{l}5 \\
5 \\
58\end{array}$ & $\begin{array}{l}\text { Female } \\
\text { Male } \\
\text { Male }\end{array}$ & $\begin{array}{l}\text { Hit on face by football } \\
\text { Toothache } \\
\text { VF cardiac arrest }\end{array}$ & $\begin{array}{l}\text { None } \\
\text { None } \\
\text { None }\end{array}$ & $\begin{array}{l}\text { Related } \\
\text { Related } \\
\text { Related }\end{array}$ & $\begin{array}{l}\text { Reassured } \\
\text { Paracetamol } \\
\text { Almost immediate BLS. Defibrillated } \\
\text { at first attempt. } \rightarrow \text { Hospital }\end{array}$ \\
\hline $5 / 2 / / 00$ & Hearts & $\begin{array}{l}16 \\
11\end{array}$ & $\begin{array}{l}\text { Female } \\
\text { Male }\end{array}$ & $\begin{array}{l}\text { Jumped off wall on way to game. Injury to left } \\
\text { os calcis } \\
\text { Vasovagal episode } \\
\text { Headache and nausea }\end{array}$ & $\begin{array}{l}\text { None } \\
\text { None }\end{array}$ & $\begin{array}{l}\text { Related } \\
\text { Related }\end{array}$ & $\begin{array}{l}\text { Reassured } \\
\text { Paracetamol }\end{array}$ \\
\hline $8 / 2 / / 00$ & Inverness CT & $\begin{array}{l}8 \\
18 \\
31\end{array}$ & $\begin{array}{l}\text { Male } \\
\text { Male } \\
\text { Female }\end{array}$ & $\begin{array}{l}\text { Thumb injury } \\
\text { Facial injury } \\
\text { Asthma }\end{array}$ & $\begin{array}{l}\text { None } \\
\text { Minor } \\
\text { None }\end{array}$ & $\begin{array}{l}\text { Related } \\
\text { Related } \\
\text { Related }\end{array}$ & $\begin{array}{l}\text { Advised to attend A\&E after game } \\
\text { Reassured } \\
\text { Salbutamol inhaler }\end{array}$ \\
\hline $1 / 3 / 00$ & Dundee & $\begin{array}{l}31 \\
14 \\
45 \\
39 \\
25 \\
32 \\
50 \\
44\end{array}$ & $\begin{array}{l}\text { Male } \\
\text { Male } \\
\text { Male } \\
\text { Male } \\
\text { Male } \\
\text { Male } \\
\text { Male } \\
\text { Female }\end{array}$ & $\begin{array}{l}\text { Agitated following bereavement } \\
\text { Abdominal pain } \\
24 \text { hour BP monitoring; cuff would not deflate } \\
\text { Eye scratch } \\
\text { Ankle sprain } \\
\text { Aches after RTA } \\
\text { Infected hand wound } \\
\text { Regular self catheterisation; had forgotten } \\
\text { catheter }\end{array}$ & $\begin{array}{l}\text { Major } \\
\text { None } \\
\text { None } \\
\text { None } \\
\text { None } \\
\text { None } \\
\text { None } \\
\text { None }\end{array}$ & $\begin{array}{l}\text { Related } \\
\text { Related } \\
\text { Related } \\
\text { Related } \\
\text { Related } \\
\text { Unrelated } \\
\text { Unrelated } \\
\text { Unrelated }\end{array}$ & $\begin{array}{l}\text { Reassurance } \\
\text { Hospital } \\
\text { Cuff removed } \\
\text { Eye pad. Chloromycetin ointment } \\
\text { Tubigrip } \\
\text { Abrasions cleaned and dressed } \\
\text { Advised to attend A\&E after match } \\
\text { No catheter available }\end{array}$ \\
\hline $8 / 3 / 00$ & Rangers & $\begin{array}{l}15 \\
18 \\
36 \\
31 \\
19 \\
38 \\
34 \\
25 \\
51 \\
39 \\
50 \\
17 \\
14 \\
50\end{array}$ & $\begin{array}{l}\text { Female } \\
\text { Female } \\
\text { Male } \\
\text { Male } \\
\text { Male } \\
\text { Male } \\
\text { Male } \\
\text { Male } \\
\text { Male } \\
\text { Male } \\
\text { Female } \\
\text { Male } \\
\text { Female } \\
\text { Female }\end{array}$ & $\begin{array}{l}\text { Low back injury after a fall } \\
\text { Fall: injury to elbow } \\
\text { Low back injury after a fall } \\
\text { Assaulted: scalp laceration } \\
\text { Fall: injury to shoulder } \\
\text { Low back injury } \\
\text { Fall: injury to back } \\
\text { Asthma } \\
\text { Chest pain; previous CABGx2 } \\
\text { Fall: head injury and laceration } \\
\text { Thigh pain following recent RTA } \\
\text { Back injury; hysterical paralysis } \\
\text { Fall: ? fractured coccyx } \\
\text { Hand laceration }\end{array}$ & $\begin{array}{l}\text { None } \\
\text { None } \\
\text { Minor } \\
\text { Minor } \\
\text { Major } \\
\text { None } \\
\text { Major } \\
\text { Minor } \\
\text { Minor } \\
\text { Major } \\
\text { Major } \\
\text { None } \\
\text { None } \\
\text { None }\end{array}$ & $\begin{array}{l}\text { Related } \\
\text { Related } \\
\text { Related } \\
\text { Related } \\
\text { Related } \\
\text { Related } \\
\text { Related } \\
\text { Related } \\
\text { Related } \\
\text { Related } \\
\text { Related } \\
\text { Related } \\
\text { Related } \\
\text { Related }\end{array}$ & $\begin{array}{l}\text { Reassured } \\
\text { ?Fracture radial head; hospital } \\
\text { Reassured } \\
\text { Reassured } \\
\text { ?Fractured clavicle; hospital } \\
\text { Reassured } \\
\text { Reassured } \\
\text { Salbutamol inhaler } \\
\text { IV access, oxygen, aspirin,; hospital } \\
\text { Wound cleaned and stapled } \\
\text { Reassured } \\
\text { Hospital } \\
\text { Hospital } \\
\text { Cleaned and dressed }\end{array}$ \\
\hline $11 / 3 / 00$ & St Johnstone & $\begin{array}{l}37 \\
66 \\
20 \\
32 \\
45\end{array}$ & $\begin{array}{l}\text { Male } \\
\text { Male } \\
\text { Male } \\
\text { Male } \\
\text { Female }\end{array}$ & $\begin{array}{l}\text { Hand laceration } \\
\text { Collapse: ?cause } \\
\text { Skin rash } \\
\text { Cellulites of leg } \\
\text { Oral swelling }\end{array}$ & $\begin{array}{l}\text { None } \\
\text { None } \\
\text { None } \\
\text { Major } \\
\text { None }\end{array}$ & $\begin{array}{l}\text { Related } \\
\text { Related } \\
\text { Related } \\
\text { Unrelated } \\
\text { Related }\end{array}$ & $\begin{array}{l}\text { Advised to attend A\&E } \\
\text { Taken by son to A\&E } \\
\text { Reassured } \\
\text { Advised to see own GP } \\
\text { Reassured }\end{array}$ \\
\hline $2 / 4 / 00$ & Kilmarnock & $\begin{array}{l}25 \\
35 \\
32 \\
24 \\
10 \\
52\end{array}$ & $\begin{array}{l}\text { Male } \\
\text { Male } \\
\text { Male } \\
\text { Female } \\
\text { Male } \\
\text { Male }\end{array}$ & $\begin{array}{l}\text { Headache } \\
\text { Head injury } \\
\text { Haemetemesis } \\
\text { Alcohol, ecstasy, and diazepam poisoning } \\
\text { Panic } \\
\text { Urticaria }\end{array}$ & $\begin{array}{l}\text { None } \\
\text { None } \\
\text { Major } \\
\text { Major } \\
\text { None } \\
\text { None }\end{array}$ & $\begin{array}{l}\text { Related } \\
\text { Related } \\
\text { Related } \\
\text { Unrelated } \\
\text { Unrelated } \\
\text { Unrelated }\end{array}$ & $\begin{array}{l}\text { Paracetamol } \\
\text { Reassured } \\
\text { Hospital } \\
\text { Left against medical advice } \\
\text { Reassured } \\
\text { Advised to consult own GP }\end{array}$ \\
\hline $5 / 4 / 00$ & Motherwell & 36 & Male & Ankle injury & None & Related & Reassured \\
\hline $15 / 4 / 00$ & Dundee & $\begin{array}{l}27 \\
19\end{array}$ & $\begin{array}{l}\text { Male } \\
\text { Female }\end{array}$ & $\begin{array}{l}\text { Ankle injury } \\
\text { SVT } 200 / \text { minute } \\
\text { Incapably drunk } \\
\text { Dizziness and nausea }\end{array}$ & $\begin{array}{l}\text { None } \\
\text { Minor }\end{array}$ & $\begin{array}{l}\text { Related } \\
\text { Related }\end{array}$ & $\begin{array}{l}\text { Ice, tubigrip } \\
\text { Successfully self treated by diver's } \\
\text { reflex } \\
\text { Returned to parents' care by police } \\
\text { Reassured }\end{array}$ \\
\hline $22 / 4 / 00$ & Hibernian & $\begin{array}{l}20 \\
58 \\
54 \\
46 \\
26 \\
17\end{array}$ & $\begin{array}{l}\text { Male } \\
\text { Male } \\
\text { Male } \\
\text { Female } \\
\text { Female } \\
\text { Female }\end{array}$ & $\begin{array}{l}\text { Chest wall pains } \\
\text { Asthma } \\
\text { Fall: severely displaced ankle fracture } \\
\text { Headache } \\
\text { Dizziness } \\
\text { "Prickly heat" }\end{array}$ & $\begin{array}{l}\text { None } \\
\text { None } \\
\text { Major } \\
\text { None } \\
\text { None } \\
\text { None }\end{array}$ & $\begin{array}{l}\text { Related } \\
\text { Related } \\
\text { Related } \\
\text { Related } \\
\text { Related } \\
\text { Unrelated }\end{array}$ & $\begin{array}{l}\text { Reassured } \\
\text { Salbutamol inhaler } \\
\text { Hospital } \\
\text { Paracetamol } \\
\text { Reassured } \\
\text { Reassured }\end{array}$ \\
\hline $6 / 5 / 00$ & Aberdeen & $\begin{array}{l}49 \\
17 \\
29 \\
9 \\
31\end{array}$ & $\begin{array}{l}\text { Male } \\
\text { Female } \\
\text { Male } \\
\text { Male } \\
\text { Female }\end{array}$ & $\begin{array}{l}\text { Unstable angina } \\
\text { Dyspepsia } \\
\text { Alcohol intoxication } \\
\text { Hand injury: hit by match ball } \\
\text { Injury to upper arm }\end{array}$ & $\begin{array}{l}\text { None } \\
\text { None } \\
\text { Major } \\
\text { None } \\
\text { None }\end{array}$ & $\begin{array}{l}\text { Related } \\
\text { Unrelated } \\
\text { Related } \\
\text { Related } \\
\text { Related }\end{array}$ & $\begin{array}{l}\text { Settled, IV access, hospital } \\
\text { Mucaine } \\
\text { Allowed home } \\
\text { "Buddy strapping" } \\
\text { Went to A\&E for } x \text { ray }\end{array}$ \\
\hline $21 / 5 / 00$ & Dundee United & $\begin{array}{l}9 \\
35\end{array}$ & $\begin{array}{l}\text { Male } \\
\text { Male }\end{array}$ & $\begin{array}{l}\text { Conjunctivitis } \\
\text { Baseball bat assault on way to game: nasal } \\
\text { fracture, facial and back injuries } \\
\text { Torticollis } \\
\text { Migraine: visual distortion }\end{array}$ & $\begin{array}{l}\text { None } \\
\text { Major }\end{array}$ & $\begin{array}{l}\text { Unrelated } \\
\text { Related }\end{array}$ & $\begin{array}{l}\text { Advice } \\
\text { Hospital } \\
\text { Paracetamol } \\
\text { Imigran, hospital }\end{array}$ \\
\hline
\end{tabular}

MI, Myocardial infarct; A\&E, Accident and Emergency; TIA, transient ischaemic attack; URTI, upper respiratory tract infection; VF, ventricular fibrillation; BLS, basic life support; BP, blood pressure; RTA, road traffic accident; CABG, coronary artery bypass graft; IV, intravenous; GP, general practitioner; SVT, supraventicular tachycardia. 


\section{Discussion}

The workload of the crowd doctors was both variable, ranging from one to 14 casualties per match, and diverse, ranging from toothache to cardiac arrest. It included surprising episodes such as two young men presenting on separate occasions with bereavement reactions, and unusual episodes such as a young man successfully treating his rapid supraventricular tachycardia by plunging his head into icy cold water. The busiest matches were the traditionally tense local derby matches with the club's city rival Rangers (fig 3). We have no doubt from our experience of previous years that the more there is at stake in a match, the busier the crowd doctors will be. The season 1999-2000 proved to be a disappointing season for Celtic resulting in reduced attendances and a subdued atmosphere. The current season has, however, started well, and we expect an increased workload if this progress is maintained.

The single cardiac arrest was less than we expected from previous seasons, with recent experiences including two cardiac arrests within ten minutes at the same match, both successfully resuscitated, and another match where successive casualties presented with cardiac arrest and dissection of an aortic aneurysm. With the availability of skilled medical care and rapid access to defibrillation, a football match is now one of the least adverse circumstances in which to have a cardiac arrest.

A cause for concern is the significant impact of alcohol intoxication on our workload. Celtic Park is a very well stewarded ground, and spectators under the influence of alcohol are not admitted. In common with other Scottish football grounds, alcohol is not on general sale. There is, however, a regrettable culture of heavy drinking in Scotland, with many consequences for local health and social services. The effects of rapid ingestion of alcohol before matches may not become apparent until after spectators have been admitted into the ground. It is of particular concern that two of the casualties affected by alcohol intoxication were children aged 14 and 15 . This is a trend that has continued since this study was completed.

The welcome social trend of football spectating becoming a family event is reflected in the relatively high numbers of women and young children requiring attention. Of note too is the proportion of casualties attending the match in a capacity other than as spectators (table 1 ). It is likely that this reflects both an awareness on their part of the facilities available, and also the responsibility on catering and stewarding supervisors to ensure that any injuries sustained by their staff at work are treated and documented.

Finally we were interested in the town of residence of the casualties, seeing this as a possible factor influencing management of the casualty. It proved to be a relevant factor in one case only, a far travelled driver who could not safely be discharged because of visual distortion due to migraine, and had to be transferred to the local accident and emergency department for observation.

We are grateful to the senior officials of Celtic Football Club for their encouragement of this article, and for the support of Dr J Mulhearn, club medical advisor, whose responsibilities include the coordination of the crowd doctoring service. We are especially grateful to our colleagues in the Scottish Ambulance Service and the St Andrew's Ambulance Association.

1 Rt Hon Lord Wheatley. Report of the inquiry into crowd safety at sports grounds. London: HMSO, 1972 (Cmnd 4952).

2 The Hillsborough Stadium Disaster: 15 April 1989. Inquiry by Lord Justice Taylor. Final Report. London: HMSO, 1990 (Cmnd 962).

3 Gibson RM. Report of the medical working party. London: HMSO, 1990

4 Kerr GW, Wilkie SC, McGuffie CA. Medical cover at Scottish football matches: have the recommendations of the Gibson Report been met? Br $\mathcal{F}$ Sports Med 1999;33: the Gibso.

5 Chesshire NJ, Gill N. An analysis of the activity of a crowd doctor at a premiership football stadium. Prehospital Immediate Care 1998;2:199-20.

\section{Take home message}

Crowd medical care at football grounds requires cooperation among first aid workers, ambulance staff, and doctors. Doctors doing this work should have appropriate training and expertise.

\section{Commentary}

Sometimes the simplest observations in life prove to be the most pertinent and interesting. This paper is of interest to all of us who provide medical cover at sporting events in Britain. Following the three major disasters in British Football and the official reports that resulted, questions have been asked about the method of appointment and qualifications of club doctors ${ }^{1}$ and the level of medical cover at sporting events. ${ }^{2}$ This paper from the medical team for Glasgow Celtic Football Club emphasises the exceptional expertise of the team, their organisation for crowd control, and the type of casualties seen.

They illustrate the increasing diversity of casualties and the opportunistic nature of those seen, and comment on the rise in female and child casualties, which we hope is a reflection of the increasing numbers attending outwith the traditional adult male spectators. 
Of most interest was the significant impact of alcohol intoxication on their workload, with almost one quarter of the casualties having alcohol as a contributory factor.

As sport becomes increasingly "professional" with spiralling financial rewards, sports governing bodies and clubs must ensure that those of us providing medical support do so with appropriate levels of training and experience. It is imperative that they provide the financial back up for this training and the provision of the medical cover that is recommended.

The days of unpaid and untrained medical support provided by doctors for the "love of the game" should be in the past-for our own sake as well as our patients.

J A MACLEAN

Department of Medicine and Therapeutics

University of Glasgow, Gardiner Institute

Western Infirmary, Glasgow G11 6NT, Scotland, UK

1 Kerr GW, Wilkie SC, McGuffie CA. Medical cover at Scottish football matches: have the recommendations of the Gibson Report been met? Br f Sports Med 1999;33:274-5.

2 Waddington I, Roderick M, Naik R. Methods of appointment and qualifications of club doctors and physiotherapists in English professional football: some problems and issues. Br f Sports Med 2001;35:48-53.

\section{British Association of Sport and Exercise Medicine in association with the National Sports Medicine Institute}

\section{Education programme 2001}

General sports medicine

23-28 September

Lilleshall Hall National Sports Centre

Practical sport and medicine meeting

4-11 October

Club La Santa, Lanzarote

Families and non-delegates welcome; booking deadline 31 July

\section{Diploma preparation course}

October

Exact date and location to be confirmed

\section{BASEM Congress}

25-28 October

Vale of Glamorgan, Wales. Further details:

Tel/Fax: +44 (0)1928 732961; email: basemoffice@compuserve.com

Injury Management and Medicine: lower limb

18-23 November

Lilleshall Hall National Sports Centre

\section{Current Concepts}

2 December

Topic, date, and location to be confirmed

For further details of these courses please contact Mr Barry Hill, The National Sports Medicine Institute, c/o Medical College of St Bartholomew's Hospital, Charterhouse Square, London EC1M 6BQ.

Tel: 02072510583 (ext 237); Fax: 0207251 0774; email: barry.hill@nsmi.org.uk

Web site: www.nsmi.org.uk 